\title{
An Eco-Friendly Paradigm for the Production of Potentially Anti-Parasitic Drugs
}

\author{
Younes Abrouki ${ }^{1,}$, , Abdelkader Anouzla ${ }^{1}$, Hayat Loukili ${ }^{1}$, Rabiaa Lotfi ${ }^{1}$, Ahmed Rayadh $^{1}$, \\ My Abdellah Bahlaoui ${ }^{1}$, Driss Zakarya ${ }^{1}$, Mohamed Zahouily ${ }^{1}$, Rachid Mamouni ${ }^{2}$, Saïd Sebti ${ }^{3}$, \\ Adil Mandil ${ }^{1}$
}

${ }^{1}$ Department of Research, Faculty of Science and Technology, University Hassan II, Mohammedia, Morocco

${ }^{2}$ Laboratory Organic Chemistry, Team Bio-Organic Chemistry Applied, Ibn Zuhr University, Agadir, Morocco

${ }^{3}$ Department of Chemistry, Faculty of Science Ben M'Sik, University Hassan II, Casablanca, Morocco

\author{
Email address: \\ abroukiyounes@yahoo.fr (Y. Abrouki)
}

\section{To cite this article:}

Younes Abrouki, Abdelkader Anouzla, Hayat Loukili, Rabiaa Lotfi, Ahmed Rayadh, My Abdellah Bahlaoui, Driss Zakarya, Mohamed Zahouily, Saïd Sebti, Rachid Mamouni. Adil Mandil. An Eco-Friendly Paradigm for the Production of Potentially Anti-Parasitic Drugs. American Journal of Environmental Protection. Special Issue: Cleaner and Sustainable Production. Vol. 4, No. 5-1, 2015 , pp. $33-36$. doi: 10.11648/j.ajeps.s.2015040501.14

\begin{abstract}
A cleaner production of various potentially anti-parasitic drugs based bis-(arylmethylidene)cycloalkanones have been developed using a heterogeneous catalysis technology in aqueous media without organic solvent. The higher activity and selectivity of our solid-state synthetic phosphate catalyst coupled with their ease of use and reduced environmental problems makes them attractive alternatives to homogeneous basic reagents.
\end{abstract}

Keywords: Green Synthesis, Heterogeneous Catalysis, Anti-Parasitic Drugs, Bis-(Arylmethylidene) Cycloalkanones, Synthetic Phosphate, Aqueous Media

\section{Introduction}

Chagas' disease and Leishmaniasis are endemic protozoan diseases recognized as epidemiological priorities by the World Health Organization (WHO). Chagas' disease is found mainly in Latin America and it is estimated to affect 12 to 14 million people. Leishmaniasis affects 12 million people in 88 countries, 72 of which are developing countries [1].

Chagas' disease [2] and Leishmaniasis [3] are caused by the trypanosomatid parasites Trypanosoma cruzi and Leishmania sp, respectively.

There are limited drugs available for the treatment of both diseases, and the currently used chemotherapeutic agents are still inadequate due to their extreme toxicity [4]. In addition, drug resistant parasite strains have become more prevalent. The search for more effective and less toxic drugs to treat both diseases have became mandatory [5].

In search for new drugs with improved antiparasitic profiles, the brazilian authors are showed that the bis-(arylmethylidene)cycloalkanones presented a activity against amastigotes forms of Trypanosoma cruzi and promastigotes forms of Leishmania amazonensis [6].
Cross-aldol condensation [7] is an important protocol for the synthesis of various bis-(arylmethylidene)cycloalkanones. This reaction is classically carried out using strong acid or base [8]. Indeed, different organometallic complexes, Lewis acid are found to be able to catalyze this reaction [9]. However, most of the above-mentioned process generated a large amount of toxic waste and involved tedious separation procedures since the reactions were homogeneous and the catalysts were destroyed or discarded on the work-up procedure [10].

Recently, because of increased environmental concerns and strong economic driver in chemical research and industry, great efforts have been made on heterogeneous catalysis, which allows the catalyst for easy isolation and recycling. Thus, solid catalysts have drawn considerable attention in organic synthesis due to their advantages such as environmental compatibility, reusability, high selectivity, simple operation, non-corrosiveness and low cost [11].

In continuation of our ongoing program to develop new environmentally process in organic synthesis, we describe in 
this paper, a environmentally friendly method for the synthesis of various bis-(arylmethylidene)cycloalkanones-based potentially anti-parasitic drugs by Cross-aldol condensation catalyzed by synthetic phosphate in aqueous media.

\section{Materials and Methods}

\subsection{Chemicals and Instrumentations}

All commercial reagents and solvents were used without further purification. X-ray diffraction (XRD) patterns of the catalysts were obtained on a Philips 1710 diffractometer using $\mathrm{Cu}-\mathrm{K} \alpha$ radiation. Surface areas were determined at $77 \mathrm{~K}$ using a Coulter SA 31000 instrument with an automated gas volumetric method employing nitrogen as the adsorbate. NMR spectra were recorded on a Bruker ARX 300 spectrometer. Mass spectra were recorded on a VG Autospec spectrometer. FTIR spectra were recorded on an ATI Mattson-Genesis Series spectrophotometer using the $\mathrm{KBr}$ disc method.

\subsection{Preparation and Characterization of Catalysts}

The fluorapatite (FAP) have been prepared by reaction between diammonium phosphate, calcium nitrate and ammonium fluoride in presence of ammonia [12]. The FAP obtained was calcined at $900^{\circ} \mathrm{C}$ before use. The structure of this catalyst was confirmed by X-ray diffraction, infrared spectra and chemical analysis. The surface area of calcined FAP was determined by the BET method and found to be $\mathrm{S}=15.4 \mathrm{~m}^{2} \mathrm{~g}^{-1}$. The total pore volume was calculated by the BJH method $\left(\mathrm{V}_{\mathrm{T}}=0.058 \mathrm{~cm}^{3} \mathrm{~g}^{-1}\right)$.

The modified fluorapatite $(\mathrm{Na} / \mathrm{FAP})$ has been prepared by impregnation of the FAP with a solution of sodium nitrate followed by calcination at $900^{\circ} \mathrm{C}$ [13]. The surface area of the new catalyst $\mathrm{Na} / \mathrm{FAP}$ was determined by the BET method as $5.4 \mathrm{~m}^{2} \mathrm{~g}^{-1}$ and the total pore volume obtained by the BJH method is $0.0032 \mathrm{~cm}^{3} \mathrm{~g}^{-1}$.

\subsection{General Procedure}

The general procedure for synthesis of bis-(arylmethylidene)cycloalkanones is reported in Figure 1, as follows: To a flask containing a mixture by taking 1:2 mole ratios of cyclopentanone 1 and arylaldehydes 2 in aqueous media, the Na/FAP catalyst was added and the mixture was stirred at reflux during 3 hours.

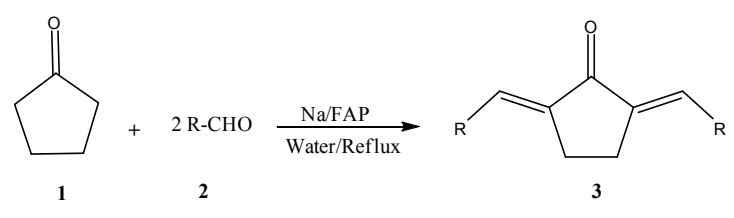

Figure 1. Synthesis of Potentially Anti-Parasitic Drugs.

Hot water $(2 \times 20 \mathrm{~mL})$ was added, followed by simple filtration. After filtration and extraction with hot water, the solution was concentrated and purified by silica gel chromatography ( $n$ hexane/ethyl acetate: 7/3). Na/FAP was reactivated by drying at $150{ }^{\circ} \mathrm{C}$ or, alternatively washed with acetone and calcined at $900{ }^{\circ} \mathrm{C}$ for $1 \mathrm{~h}$. The crude product 3 was identified by melting point, ${ }^{1} \mathrm{HNMR},{ }^{13} \mathrm{C} \mathrm{NMR}$, and IR spectroscopies.

\section{Results and Discussion}

After synthesis and characterization of fluorapatite and $\mathrm{Na} / \mathrm{FAP}$, we tested these two materials as catalysts in the synthesis of bis-(arylmethylidene)cycloalkanones in aqueous media, which tends to a green synthesis strategy, going from modernization of organic synthesis toward sustainability. The cross-aldol condensation between cyclopentanone 1 and benzaldehyde $2 \mathrm{a}$, were chosen as model substrates to determine suitable reaction conditions for synthesis of bis-(arylmethylidene)cycloalkanones. The optimal reaction condition for synthesis of this product was obtained by central composite design [14] and it's reported in Figure 2.

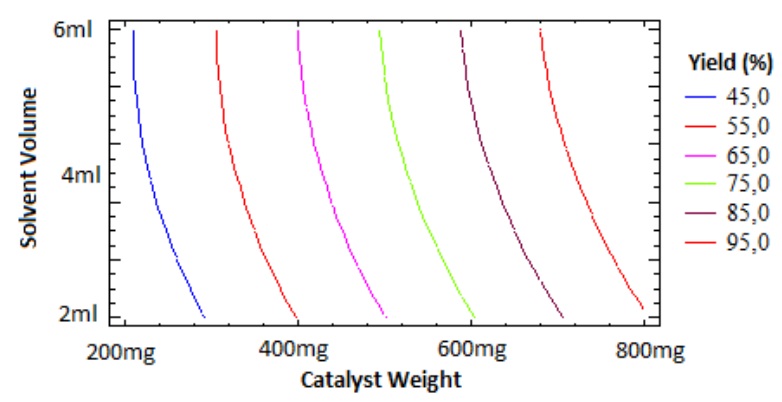

Figure 2. Optimization of reaction condition for cross-aldol condensation by central composite design.

We studied the influence of several parameters on a model cross-aldol condensation, specifically: (i) optimal catalyst preparation conditions ( $\mathrm{Na} / \mathrm{FAP})$, (ii) quantity of catalyst, and (iii) kinetics of the reaction.

The investigation of central composite design showed that the optimum model reaction conditions are: catalyst weight $=$ $700 \mathrm{mg}$, reaction time $=3 \mathrm{~h}$ and solvent volume $=5 \mathrm{~mL}$. To determine the scope and limitation of this reaction, the optimum condensations were applied to other substrates as shown in table 1 .

Table 1. Synthesis of various bis-(arylmethylidene)cycloalkanones.

\begin{tabular}{|c|c|c|c|}
\hline Entry & R-CHO & Product $\left({ }^{*}\right)$ & $\begin{array}{l}\text { Yield } \\
(\%)\end{array}$ \\
\hline $3 a$ & $\mathrm{C}_{6} \mathrm{H}_{5} \mathrm{CHO}$ & & 92 \\
\hline $3 b$ & $4-\mathrm{Cl}-\mathrm{C}_{6} \mathrm{H}_{4} \mathrm{CHO}$ & & 83 \\
\hline $3 \mathrm{c}$ & 4-MeO- $\mathrm{C}_{6} \mathrm{H}_{4} \mathrm{CHO}$ & & 72 \\
\hline
\end{tabular}






* The analytical data were identical to those reported in the literature.

In this regard, the preparation of potentially anti-parasitic drugs was carried out under reflux conditions by taking $1: 2$ mole ratios of cyclopentanones and arylaldehydes in water in the presence of modified fluorapatite $\mathrm{Na}$ /FAP catalyst to give the desired bis-(arylmethylidene)cycloalkanones in excellent yields. In general, the use of FAP as the heterogeneous catalyst in the crossed-aldol condensation has allowed the isolation of our products in low yields with slow reaction time. Under similar conditions in the absence of synthetic phosphate FAP or $\mathrm{Na} / \mathrm{FAP}$ catalyst, only the starting material was regenerated, thus highlighting the role of this catalyst. When the reactions were examined in different solvents such as methanol, toluene, chloroform, water and THF, we found that water is the best solvent due to high yields and shorter reaction times. Moreover, using water as the reaction medium made the separation process much easier with high purity of final product than using organic solvents. However, when toluene, THF, chloroform, and methanol were used as solvents, a homogenous mixture of product and starting materials was obtained at the end of the reaction. After filtration of the final product, synthetic phosphate catalyst was recovered quantitatively by simple filtration and regenerated by calcination for each new reuse. The table show that the comparison of the results obtained by our method with some of those reported shows the efficiency of this method because of shorter reaction times, high yields, and application of an inexpensive and readily available catalyst. A simple procedure and the use of water as a solvent are expected to contribute to the development of more benign crossed-aldol reactions. The modified synthetic phosphate $\mathrm{Na} / \mathrm{FAP}$ catalyst is a new, inexpensive, and attractive solid support which can contribute to the development of catalytic processes and reduced environmental problems and it is the first use of this material as the catalyst.

\section{Conclusions}

In conclusion, we have developed a clean and easy method for the synthesis of potentially anti-parasitic drugs based bis-(arylmethylidene)cycloalkanones using an inexpensive, reusable, easy to handle, noncorrosive, and environmentally benign catalysts, fluorapatite and especially sodium-modified fluorapatite. The use of these catalysts offers diverse advantages including simplicity of operation due to the heterogeneous nature of reaction, easy workup, high yields, and catalyst reusability.

\section{References}

[1] WHO, World Health Organization. Available from: $\mathrm{http}: / /$ apps.who.int/tdr/svc/publications/tdrnews/tdr-news-issu e-84 (accessed 02.22.10).

[2] J.A. Urbina, Parasitological cure of Chagas disease, Oswaldo Cruz. 94 (1999) 349-355.

[3] S.A. Grevelink, E.A. Lerner, Leishmaniasis, J. Am. Acad. Dermatol. 34 (1996) 257-272.

[4] S.L. Croft, G.H. Coombs, Leishmaniasis - current chemotherapy and recent advances in the search for novel drugs, Trends Parasitol. 19 (2003) 502-508.

[5] R.B. Oliveira, A.B.M. Vaz, R.O. Alves, D.B. Liarte, C.L. Donnici, A.J. Romanha, C.L. Zani, Arylfurans as potential Trypanosoma cruzi trypanothione, Mem. Inst. Oswaldo Cruz 101 (2006) 169-173.

[6] F.P. Braga, V.P. Alves, R.S. Ferreira, R.B. Fradico, P.S. Lage, M.C. Duarte, T.G. Ribeiro, A.S. Júnior, A.J. Romanha, M.L. Tonini, M. Steindel, E.F. Coelho, R.B. Oliveira, Synthesis and evaluation of the antiparasitic activity of bis-(arylmethylidene) cycloalkanones, European Journal of Medicinal Chemistry 71 (2014) 282-289.

[7] Y. Riadi, R. Mamouni, R. Azzalou, R. Boulahjar, Y. Abrouki, M. El Haddad, S. Routier, G. Guillaumet, S. Lazar, Animal bone meal as an efficient catalyst for crossed-aldol condensation, Tetrahedron Letters 51 (2010) 6715-6717

[8] P. Salehi, M. Dabiri, M.A. Zolfigol, M.B. Fard, Silica sulfuric acid as an efficient and reusable reagent for crossed-aldol condensation of ketones with aromatic aldehydes under solvent-free conditions, J. Braz. Chem. Soc. 15 (2004) 773-776.

[9] M.S. Abaee, M.M. Mojtahedi, S. Forghani, N.M. Ghandchi, M. Forouzani, R. Sharifi, B. Chaharnazm, A Green, inexpensive and efficient organocatalyzed procedure for aqueous aldol condensations, J. Braz. Chem. Soc. 20 (2009) 1895-1900.

[10] M.S. Abaee, M.M. Mojtahedi, R. Sharifi, M.M. Zahedi, H. Abbasi, K. Tabar- Heidar, Facile synthesis of bis(arylmethylidene)cycloalkanones mediated by lithium perchlorate under solvent-free conditions, Iran. J. Chem. Soc. 3 (2006) 293-296.

[11] Y. Abrouki, M. Zahouily, A. Rayadh, B. Bahlaouan, S. Sebti, A natural phosphate and doped-catalyzed Michael addition of mercaptans to $\alpha, \beta$-unsaturated carbonyl compounds, Tet. Lett., 43, (2002), pp. 8951-8953.

[12] M. Zahouily, Y. Abrouki, A. Rayadh, S. Sebti, H. Dhimane, M. David, Fluorapatite: efficient catalyst for the Michael addition, Tet. Lett., 44 (2003), 2463-2465.

[13] A Solhy, A Smahi, H El Badaoui, B Elaabar, A. Amoukal, A. Tikad, S. Sebti, D.J. Macquarrie, Efficient hydration of nitriles to amides catalysed by sodium nitrate modified fluorapatite, Tet. Lett., 44 (2003), 4031-4033. 
[14] Y. Abrouki, A. Anouzla, H. Loukili, R. Lotfi, A. Rayadh, M.A. Bahlaoui, S. Sebti, D. Zakarya, M. Zahouily, Central composite experimental design applied to the catalytic carbon-sulfur bond formation by fluorapatite catalyst, American Journal of Applied Chemistry, 1 (2013), 22-27. 